\title{
THE PARABOLIC LOGISTIC EQUATION WITH BLOW-UP INITIAL AND BOUNDARY VALUES
}

\author{
YIHONG DU, RUI PENG AND PETER POLÁČIK
}

ABSTRACT. In this article, we investigate the parabolic logistic equation with blow-up initial and boundary values over a smooth bounded domain $\Omega$ :

$$
\begin{cases}u_{t}-\Delta u=a(x, t) u-b(x, t) u^{p} & \text { in } \Omega \times(0, T), \\ u=\infty & \text { on } \partial \Omega \times(0, T) \cup \bar{\Omega} \times\{0\},\end{cases}
$$

where $T>0$ and $p>1$ are constants, $a$ and $b$ are continuous functions, with $b>0$ in $\Omega \times$ $[0, T), b(x, T) \equiv 0$. We study the existence and uniqueness of positive solutions, and their asymptotic behavior near the parabolic boundary. Under the extra condition that $b(x, t) \geq$ $c(T-t)^{\theta} d(x, \partial \Omega)^{\beta}$ on $\Omega \times[0, T)$ for some constants $c>0, \theta>0$ and $\beta>-2$, we show that such a solution stays bounded in any compact subset of $\Omega$ as $t$ increases to $T$, and hence solves the equation up to $t=T$.

\section{INTRODUCTION}

In this work, we study the parabolic logistic equation with blow-up initial and boundary values:

$$
\begin{cases}u_{t}-\Delta u=a(x, t) u-b(x, t) u^{p} & \text { in } \Omega \times(0, T), \\ u=\infty & \text { on } \partial \Omega \times(0, T), \\ u=\infty & \text { on } \bar{\Omega} \times\{0\},\end{cases}
$$

where $\Omega \subset \mathbb{R}^{N}(N \geq 1)$ is a bounded smooth domain, $T>0$ and $p>1$ are constants, $a(x, t)$ and $b(x, t)$ are continuous functions on $\Omega \times[0, T]$, with $b>0$ in $\Omega \times[0, T)$ and $b=0$ on $\Omega \times\{T\}$.

Throughout this work, by $u=\infty$ on $\partial \Omega \times(0, T)$, we mean that

$$
u(x, t) \rightarrow \infty \text { as } d(x, \partial \Omega) \rightarrow 0 \text { uniformly for } t \in(0, T),
$$

Date: February 1, 2012.

1991 Mathematics Subject Classification. 35K20, 35K60, 35B30, 35B40.

Key words and phrases. Parabolic logistic equation, initial and boundary blow up, positive solution, uniqueness, asymptotic behavior.

Y. Du: Department of Mathematics, School of Science and Technology, University of New England, Armidale, NSW 2351, Australia. Email: ydu@turing.une.edu.au.

R. Peng: Department of Mathematics and Statistics, Memorial University of Newfoundland, St. John's, NL, A1C 5S7, Canada. Email: pengrui_seu@163.com.

P. Poláčik: Department of Mathematics, University of Minnesota, Minneapolis, MN 55455, USA. Email: polacik@math.umn.edu.

Part of this work was carried out during a visit of Y. Du to the University of Minnesota and a visit of P. Poláčik to the University of New England. Y. Du was partially supported by the Australian Research Council, R. Peng was partially supported by the National Natural Science Foundation of China 10801090, 10871185, 10771032, and P. Poláčik was partially supported by NSF Grant DMS-0900947. 
and by $u=\infty$ on $\bar{\Omega} \times\{0\}$, we mean that

$$
u(x, t) \rightarrow \infty \text { as } t \rightarrow 0 \quad \text { uniformly for } x \in \bar{\Omega} .
$$

We are interested in the existence and uniqueness of positive solutions to (1.1), and the behavior of the solutions near $t=T$ and near the parabolic boundary

$$
\Sigma(T):=\partial \Omega \times(0, T) \cup \Omega \times\{0\} .
$$

Our investigation in the present work was partly motivated by [8] concerning the longtime asymptotic behavior of the solution of a parabolic logistic equation with a degenerate spatial-temporal coefficient $b(x, t)$, of the form

$$
\begin{cases}v_{t}-\Delta v=a v-b(x, t) v^{p} & \text { in } D \times(0, \infty), \\ \partial_{\nu} v=0 & \text { on } \partial D \times(0, \infty), \\ v(x, 0)=v_{0}(x) \geq, \not \equiv 0 & \text { in } D,\end{cases}
$$

where $a$ is a constant (for simplicity), $p>1, b(x, t)$ is Hölder continuous and $L$-periodic in $t$, and there exist Hölder continuous functions $p(x)$ and $q(t)$ such that

$$
c_{1} p(x) q(t) \leq b(x, t) \leq c_{2} p(x) q(t) \quad\left(x \in D, t \in \mathbb{R}^{1}\right)
$$

for some positive constants $c_{1}$ and $c_{2}, p(x)>0$ in a smooth domain $\Omega$ satisfying $\bar{\Omega} \subset D$, $p(x) \equiv 0$ in $D \backslash \Omega$, and $q(t)$ is $L$-periodic and satisfies, for some $T \in(0, L)$,

$$
q(t)>0 \text { in }(0, T), q(t) \equiv 0 \text { in }[T, L] .
$$

In [8], the complementary case that $p(x)>0$ in $D \backslash \bar{\Omega}$ and $p(x) \equiv 0$ in $\bar{\Omega}$ was studied, but the results there carry over to the current case $p(x) \equiv 0$ in $D \backslash \Omega, p(x)>0$ in $\Omega$ with only minor variations of the proofs. These results indicate that there exists $a^{*}>0$ such that when $a \geq a^{*}$, the unique positive solution $v(x, t)$ of $(1.2)$ satisfies

$$
\lim _{n \rightarrow \infty} v(x, t+n L)=\infty
$$

uniformly for $(x, t)$ in compact subsets of $\{(\bar{D} \backslash \bar{\Omega}) \times[0, T]\} \cup\{\bar{D} \times(T, L]\}$, and

$$
\lim _{n \rightarrow \infty} v(x, t+n L)=u(x, t)
$$

uniformly for $(x, t)$ in compact subsets of $\Omega \times[0, T)$, where $u$ is the minimal positive solution of $(1.1)$ with $a(x, t) \equiv$ constant.

In order to see the point clearer, we restate the above conclusions from a slightly different angle. In any compact subset of the infinite cylinder $(\bar{D} \backslash \Omega) \times \mathbb{R}^{1}$, clearly $\lim _{n \rightarrow \infty} v(x, t+n L)=\infty$ uniformly. In the infinite cylinder $\Omega \times \mathbb{R}^{1}$, we define

$$
u^{*}(x, t)= \begin{cases}u(x, t), & (x, t) \in \Omega \times(0, T), \\ \infty, & (x, t) \in \Omega \times(T, L],\end{cases}
$$

and extend $u^{*}$ periodically into $\Omega \times\left(\mathbb{R}^{1} \backslash S\right), S:=\{T+k L: k=0, \pm 1, \pm 2, \ldots\}$. Then

$$
\lim _{n \rightarrow \infty} v(x, t+n L)=u^{*}(x, t)
$$


uniformly for $(x, t)$ in compact subsets of $\Omega \times\left(\mathbb{R}^{1} \backslash S\right)$. Thus the behavior of $\lim _{n \rightarrow \infty} v(x, t+$ $n L)$ is unclear only over the set $\Omega \times S$.

To answer this remaining question, one needs to know the asymptotic behavior of the solutions to (1.1) near $t=T$. The analogous results of [8] reveal that (1.1) has a maximal and minimal solution, but the questions of uniqueness and asymptotic behavior of the solution near $t=T$ and near the parabolic boundary were left open.

These questions will be addressed here under the following assumptions on $a(x, t)$ and $b(x, t)$ :

$$
\left\{\begin{array}{l}
a \text { and } b \text { are continuous in } \bar{\Omega} \times[0, T] \text { and } \Omega \times[0, T], \text { respectively, } \\
b(x, t)>0 \text { in } \Omega \times[0, T), \quad b(x, t)=0 \text { on } \bar{\Omega} \times\{T\}
\end{array}\right.
$$

and there exist a constant $\beta>-2$ and positive continuous functions $\alpha_{1}(t)$ and $\alpha_{2}(t)$ on $[0, T)$ such that

$$
\alpha_{1}(t) d(x, \partial \Omega)^{\beta} \leq b(x, t) \leq \alpha_{2}(t) d(x, \partial \Omega)^{\beta} \text { for }(x, t) \in \Omega \times[0, T) .
$$

We note that, due to (1.4), necessarily $\alpha_{1}(T)=0$. Note also that if $\beta \leq-2$, then (1.1) may not have a solution. Indeed, if $u(x, t)$ satisfies

$$
u_{t}-\Delta u=a(x, t) u-d(x, \partial \Omega)^{\beta} u^{p} \text { in } \Omega \times(0, T),
$$

with $\beta \leq-2$, then one can easily modify the arguments in [4] or Lemma 6.12 of [6] (where the corresponding elliptic problem was considered) to show that for each fixed $t \in(0, T)$, $u(x, t) \leq C(t) d(x, \partial \Omega)^{-\frac{2+\beta}{p-1}}$ for all $x$ near $\partial \Omega$. Hence in such a case $u(x, t)$ is always bounded from above for $x$ near $\partial \Omega$.

The first two results in this paper are concerned with the behavior of positive solutions of (1.1) near $t=0$ and near $t=T$.

Theorem 1.1. Under conditions (1.4) and (1.5), problem (1.1) has a maximal positive solution $\bar{u}$ and a minimal positive solution $\underline{u}$, in the sense that any positive solution $u$ of (1.1) satisfies $\underline{u} \leq u \leq \bar{u}$. Moreover, for any given $t_{0} \in(0, T)$, there exist positive constants $c_{1}$ and $c_{2}$, depending on $t_{0}$, such that

$$
\underline{u}(x, t) \geq c_{1}\left(t^{-\frac{1}{\bar{p}_{\beta}-1}}+d(x, \partial \Omega)^{-\frac{2+\beta}{p-1}}\right) \quad\left((x, t) \in \Omega \times\left(0, t_{0}\right]\right)
$$

and

$$
\bar{u}(x, t) \leq c_{2}\left(t^{-\frac{1}{\underline{p}^{-1}}}+d(x, \partial \Omega)^{-\frac{2+\beta}{p-1}}\right) \quad\left((x, t) \in \Omega \times\left(0, t_{0}\right]\right)
$$

where

$$
\bar{p}_{\beta}=\max \left\{p, \frac{2 p+\beta}{2+\beta}\right\}, \underline{p}_{\beta}=\min \left\{p, \frac{2 p+\beta}{2+\beta}\right\} .
$$

Clearly

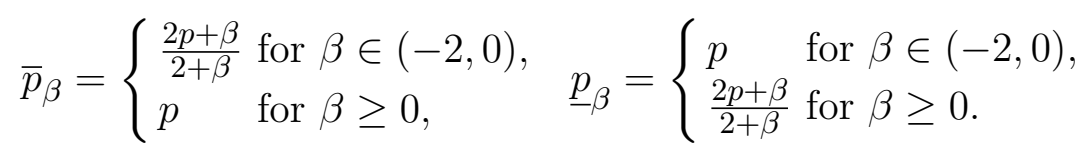


Theorem 1.2. Under the conditions of Theorem 1.1, if we further assume that $\alpha_{1}(t) \geq c_{0}(T-t)^{\theta}$ in $[0, T)$ for some positive constants $c_{0}$ and $\theta$, then for any $t_{0} \in(0, T)$, there exists $C=C\left(t_{0}\right)>0$ such that

$$
\text { 8) } \bar{u}(x, t) \leq C \min \left\{(T-t)^{-\frac{\theta}{p-1}}, d(x, \partial \Omega)^{-\frac{2 \theta}{p-1}}\right\} d(x, \partial \Omega)^{-\frac{2+\beta}{p-1}} \quad\left((x, t) \in \Omega \times\left[t_{0}, T\right)\right) .
$$

As we will see below, the behavior of the positive solutions of (1.1) near $t=0$ will help to determine whether the solution is unique. Theorem 1.2 implies that under the extra mild condition imposed there, the asymptotic limit $u^{*}(x, t)$ of the solution $v(x, t)$ of (1.2) extends to a continuous function in $\Omega \times(k L, k L+T]$ for every integer $k$, and hence exhibits an infinite jump each time $t$ increases across $k L+T$.

Moreover, let us mention, without elaborating, that Theorem 1.2 can also be used to handle the problem (1.2), where (1.3) is satisfied with $q(t)>0$ except at finitely many points in $(0, L]$. If $q(t)$ satisfies the condition in Theorem 1.2 at each of its vanishing points, then one can use Theorem 1.2 to show that, there exists $\tilde{a}^{*}>0$ so that for $a \geq \tilde{a}^{*}$, the unique positive solution $v(x, t)$ of (1.2) satisfies $\lim _{t \rightarrow \infty} v(x, t)=\infty$ uniformly in $\bar{D} \backslash \Omega$, and $\lim _{n \rightarrow \infty} v(x, t+n L)=U(x, t)$ uniformly in any compact subset of $\Omega \times \mathbb{R}^{1}$, where $U$ is the minimal positive solution of

$$
U_{t}-\Delta U=a U-b(x, t) U^{p} \text { in } \Omega \times \mathbb{R}^{1}, u=\infty \text { on } \partial \Omega \times \mathbb{R}^{1},
$$

which is $L$-periodic in $t$.

The other results of this paper deal with the uniqueness and the local behavior of the solution of (1.1) near the parabolic boundary $\Sigma(T)$. These questions were considered in [3] for the (spatially and temporally) autonomous problem

$$
u_{t}-\Delta \phi(u)=f(u) \text { in } \Omega \times(0, \infty), u=\infty \text { on } \Sigma,
$$

under suitable conditions on the functions $\phi$ and $f$, where $\Sigma:=\partial \Omega \times(0, \infty) \cup \Omega \times\{0\}$.

If we take $\phi(u)=u$ and $f(u)=a_{0} u-b_{0} u^{p}$ with constants $p>1, a_{0}>0, b_{0}>0$, then the result of [3] implies that

(i) $u(x, t) / t^{-1 /(p-1)} \rightarrow\left[(p-1) b_{0}\right]^{-1 /(p-1)} \quad$ as $t \rightarrow 0 \quad(x \in \Omega)$,

(ii) $U(x, t) / d(x, \partial \Omega)^{-2 /(p-1)} \rightarrow\left[\frac{2(p+1)}{b_{0}(p-1)^{2}}\right]^{1 /(p-1)}$ as $x \rightarrow \partial \Omega \quad(t>0)$.

Indeed, (i) and (ii) above are consequences of the fact that

$$
u(x, t) / z(t) \rightarrow 1 \text { as } t \rightarrow 0 \text { for fixed } x \in \Omega,
$$

and

$$
u(x, t) / V(x) \rightarrow 1 \text { as } x \rightarrow \partial \Omega \text { for fixed } t>0,
$$

where $z(t)$ is the unique solution of the ODE

$$
z^{\prime}=a_{0} z-b_{0} z^{p} \text { for } t>0, z(0)=\infty,
$$

and $V(x)$ is the unique solution to the elliptic boundary blow-up problem

$$
-\Delta V=a_{0} V-b_{0} V^{p} \text { in } \Omega,\left.V\right|_{\partial \Omega}=\infty .
$$


Related results for nonsmooth domains can be found in $[1,2,11]$. For the non-autonomous problem (1.1), we have the following results.

Theorem 1.3. Under the assumptions of Theorem 1.1, let $u(x, t)$ be any solution of (1.1). Then
(i) $\frac{u(x, t)}{t^{-1 /(p-1)}} \rightarrow[(p-1) b(x, 0)]^{-1 /(p-1)}$
as $t \rightarrow 0$ for fixed $x \in \Omega$;
(ii) $\frac{u(x, t)}{d(x, \partial \Omega)^{-(2+\beta) /(p-1)}} \rightarrow\left[\frac{2(p+1)}{\gamma\left(x_{0}, t\right)(p-1)^{2}}\right]^{1 /(p-1)}$ as $x \rightarrow x_{0} \in \partial \Omega$ for

fixed $t \in(0, T)$, provided that

$$
\gamma(x, t):=\frac{b(x, t)}{d(x, \partial \Omega)^{\beta}}
$$

extends to a continuous function on $\bar{\Omega} \times(0, T)$.

Theorem 1.4. Under the assumptions of Theorem 1.1, if $\beta=0$, then (1.1) has a unique positive solution.

Theorems 1.1 and 1.2 are proved in section 2. The proof of Theorem 1.1 is based on various comparison arguments, while that for Theorem 1.2 is based on a scaling argument. We remark that the scaling argument here is different from those in [12], where a suitable scaling process based on a key doubling lemma is combined with a Liouville type result to deduce some universal a priori bounds; our proof here does not rely on such a lemma or a Liouville theorem, instead it combines a suitable scaling process with a comparison argument to obtain the required bound.

In section 3, we prove Theorems 1.3 and 1.4. Since (1.1) has variable coefficients, and $b(x, t)$ may vanish or blow-up on $\partial \Omega$, we need to introduce some new localization techniques to prove Theorem 1.3, which are considerably different from the arguments in [3], where only constant coefficients appear in the equation. The equations in $[1,2,11]$ also only involve constant coefficients. Theorem 1.4 follows from Theorem 1.1 and a convex function technique of Marcus and Véron [9, 10].

\section{Estimates NEAR $t=0$ AND NEAR $t=T$}

We prove Theorems 1.1 and 1.2 in this section. To simplify notations, we will write $d(x)=d(x, \partial \Omega)$ from now on.

Proof of Theorem 1.1: We first prove the existence of a minimal positive solution $\underline{u}(x, t)$ and a maximal positive solution $\bar{u}(x, t)$ of $(1.1)$ in the sense that any positive solution $u(x, t)$ of $(1.1)$ satisfies $\underline{u}(x, t) \leq u(x, t) \leq \bar{u}(x, t)$ in $\Omega \times(0, T)$. The proof of $(1.6)$ and (1.7) partly builds upon the arguments leading to the existence of the minimal and maximal solutions.

For arbitrarily small $\epsilon>0$, since $\alpha_{1}(t)>0$ in $[0, T-\epsilon]$, we may assume that $\alpha_{1}(t) \geq m_{\epsilon}$ on $[0, T-\epsilon]$ for some positive constant $m_{\epsilon}$. Choose $A>0$ such that $|a(x, t)| \leq A$ in 
$\Omega \times(0, T]$. Then, for any given constant $n \geq 1$, we consider the problem

$$
\begin{cases}u_{t}-\Delta u=a u-b u^{p} & \text { in } \Omega \times(0, T-\epsilon), \\ u=n & \text { on } \partial \Omega \times(0, T-\epsilon) \cup \bar{\Omega} \times\{0\} .\end{cases}
$$

It is clear that (2.1) has a unique positive solution $u_{n}$ for any given $n \geq 1, \epsilon>0$. Moreover, the classical comparison theorem for parabolic equations guarantees that $u_{n}$ is strictly increasing in $n$, that is, $u_{n}<u_{n+1}$ on $\bar{\Omega} \times[0, T-\epsilon]$.

In the sequel, we will find a supersolution of (2.1), which is independent of $n$. For this purpose, we consider the following two auxiliary problems:

$$
w^{\prime}=A w-w^{\underline{p}} \quad \text { for } t>0, \quad w(0)=\infty,
$$

and

$$
-\Delta z=A z-d(x)^{\beta} z^{p} \quad \text { in } \Omega, \quad z=\infty \text { on } \partial \Omega .
$$

The unique solution $w^{*}$ of $(2.2)$ can be explicitly written as

$$
w^{*}(t)=A^{\frac{1}{\underline{p}^{-1}}} e^{A t}\left[e^{A\left(\underline{p}_{\beta}-1\right) t}-1\right]^{\frac{1}{1-\underline{\underline{p}} \beta}}, \quad t>0 .
$$

It is well-known (see, for example, Theorem 6.15 in [5]) that problem (2.3) admits a unique positive solution $z^{*}$, and

$$
\lim _{x \rightarrow \partial \Omega} \frac{z^{*}(x)}{d(x)^{-\frac{2+\beta}{p-1}}}=\left[\frac{(2+\beta)(1+\beta+p)}{(p-1)^{2}}\right]^{\frac{1}{p-1}} .
$$

It follows that

$$
c_{1} d(x)^{-\frac{2+\beta}{p-1}} \leq z^{*}(x) \leq c_{2} d(x)^{-\frac{2+\beta}{p-1}} \quad(x \in \Omega)
$$

for some positive constants $c_{1}$ and $c_{2}$.

If $\beta \in(-2,0)$, then we can find $c_{3}>0$ such that $c_{3} d(x)^{\beta} \geq 1$ in $\Omega$. Recalling also that $\underline{p}_{\beta}=p$ in this case, it follows that

$$
\left(w^{*}\right)^{\prime} \geq A w^{*}-c_{3} d(x)^{\beta}\left(w^{*}\right)^{p} \quad(x \in \Omega, t>0) .
$$

Thus we can find $M>1$ sufficiently large such that $U^{*}:=M\left(w^{*}+z^{*}\right)$ satisfies

$$
U_{t}^{*}-\Delta U^{*} \geq A U^{*}-m_{\epsilon} d(x)^{\beta}\left(U^{*}\right)^{p} \quad(x \in \Omega, t>0) .
$$

It follows that

$$
U_{t}^{*}-\Delta U^{*} \geq a(x, t) U^{*}-b(x, t)\left(U^{*}\right)^{p} \quad(x \in \Omega, t \in(0, T-\epsilon]) .
$$

If $\beta \geq 0$, then there exists $c_{4}>0$ such that $d(x)^{\beta} \leq c_{4} z^{*}(x)^{-\beta \frac{p-1}{2+\beta}}$ and thus

$$
-\Delta z^{*} \geq A z^{*}-c_{4}\left(z^{*}\right)^{\underline{p}}
$$

Thus, we can find $M_{0} \geq 1$ large enough such that $U_{0}=M_{0}\left(w^{*}+z^{*}\right)$ satisfies

$$
\left(U_{0}\right)_{t}-\Delta U \geq A U_{0}-U_{0}^{\underline{p} \beta} \quad(x \in \Omega, t>0) .
$$


Since

$$
\underline{p}_{\beta}=p-\beta \frac{p-1}{2+\beta} \leq p
$$

and

$$
U_{0} \geq z^{*} \geq c_{1} d(x)^{-\frac{2+\beta}{p-1}}
$$

we have

$$
U_{0}^{\underline{p}_{\beta}} \leq c_{5} d(x)^{\beta} U_{0}^{p}
$$

for some $c_{5}>0$ and thus

$$
\left(U_{0}\right)_{t}-\Delta U_{0} \geq A U_{0}-c_{5} d(x)^{\beta} U_{0}^{p} \quad(x \in \Omega, \forall t>0) .
$$

It follows that there exists $M \geq M_{0}$ such that $U^{*}=M\left(w^{*}+z^{*}\right)=\left(M / M_{0}\right) U_{0}$ satisfies $(2.5)$.

For any fixed $n$, we have $U^{*}(x, t)>u_{n}(x, t)$ on $\partial \Omega \times(0, T-\epsilon)$ and on $\bar{\Omega}$ for all small $t>0$. Hence, for all $n \geq 1$, by the comparison principle, we have $u_{n}(x, t) \leq U^{*}(x, t)$ on $\bar{\Omega} \times(0, T-\epsilon]$. It should be noticed that, for fixed small $\epsilon>0$ and any compact subset $K$ of $\Omega, U^{*}$ is bounded on $K \times[\epsilon, T-\epsilon]$. As a consequence, by standard regularity arguments, $u_{n}(x, t) \rightarrow \underline{u}(x, t)$ as $n \rightarrow \infty$ uniformly on any compact subset of $\Omega \times(0, T)$, where $\underline{u}$ satisfies (1.1). Thus, $\underline{u}$ is a solution to (1.1). (The fact that the boundary conditions are satisfied by $\underline{u}$ can be easily proved as in the elliptic case; see e.g. page 13 of [7]. This also follows from the proof of (1.6) given below.) Actually, $\underline{u}$ is the minimal positive solution of (1.1). Indeed, let $u$ be any positive solution of (1.1). Then, for any small $\epsilon>0$, we can easily apply the parabolic comparison principle to conclude that $u_{n}<u$ in $\Omega \times(0, T-\epsilon)$. Letting $n \rightarrow \infty$ we deduce $\underline{u} \leq u$ on $\bar{\Omega} \times(0, T-\epsilon]$. By the arbitrariness of $\epsilon, \underline{u} \leq u$ in $\bar{\Omega} \times(0, T)$, which implies that $\underline{u}$ is the minimal positive solution.

We next prove the existence of a maximal positive solution of (1.1). To achieve this, for any small $\epsilon>0$, we define $\Omega_{\epsilon}=\{x \in \Omega: d(x, \partial \Omega)>\epsilon\}$. Obviously, for small $\epsilon, \partial \Omega_{\epsilon}$ has the same smoothness as $\partial \Omega$. Then, we consider the following problem:

$$
\begin{cases}u_{t}-\Delta u=a(x, t) u-b(x, t) u^{p} & \text { in } \Omega_{\epsilon} \times(\epsilon, T), \\ u=\infty & \text { on } \partial \Omega_{\epsilon} \times(\epsilon, T) \cup \bar{\Omega}_{\epsilon} \times\{\epsilon\} .\end{cases}
$$

Let us denote by $\underline{u}^{\epsilon}$ the minimal positive solution of (2.6).

By using the parabolic comparison principle, we easily deduce that $\underline{u}^{\epsilon_{1}} \geq \underline{u}^{\epsilon_{2}} \geq \underline{u}$ when $\epsilon_{1}>\epsilon_{2}>0$. Therefore, one can extract a decreasing sequence $\epsilon_{n}$ satisfying $\epsilon_{n} \rightarrow 0$, such that $\underline{u}^{\epsilon_{n}} \rightarrow \bar{u}$ as $\epsilon_{n} \rightarrow 0$ and $\bar{u}$ solves (1.1). Moreover, $\bar{u}$ is the maximal positive solution of (1.1). Indeed, for any positive solution $u$ of (1.1), it follows from the parabolic comparison principle again that $\underline{u}^{\epsilon_{n}}>u$ in $\Omega_{\epsilon_{n}} \times\left(\epsilon_{n}, T\right)$ for each $n$. By taking $n \rightarrow \infty$ we obtain $\bar{u} \geq u$. Hence $\bar{u}$ is the maximal positive solution of (1.1).

Finally, we prove (1.6) and (1.7). By checking the previous analysis, we have

$$
\underline{u} \leq \bar{u} \leq M\left(w_{\delta}^{*}+z_{\delta}^{*}\right) \text { in } \Omega_{\delta} \times(\delta, T-\epsilon),
$$


where $M \geq 1$ is independent of $\delta, w_{\delta}^{*}(t)=w^{*}(t-\delta)$ and $z_{\delta}^{*}$ is the unique positive solution of

$$
-\Delta z=A z-d(x)^{\beta} z^{p} \text { in } \Omega_{\delta}, z=\infty \text { on } \partial \Omega_{\delta}
$$

for the case $\beta \in(-2,0)$, and $z_{\delta}^{*}$ is the the unique positive solution of

$$
-\Delta z=A z-d\left(x, \partial \Omega_{\delta}\right)^{\beta} z^{p} \text { in } \Omega_{\delta}, z=\infty \text { on } \partial \Omega_{\delta}
$$

for the case $\beta \geq 0$. (The existence and uniqueness of $z_{\delta}^{*}$ in both cases are well-known; see, for example, Theorem 6.15 of [6].) Letting $\delta \rightarrow 0$ in (2.7), and using the easily proved fact that $z_{\delta}^{*} \rightarrow z^{*}$, we deduce

$$
\underline{u} \leq \bar{u} \leq M\left(w^{*}+z^{*}\right) \text { in } \Omega \times(0, T-\epsilon) .
$$

For any given $t_{0} \in(0, T)$, we may choose $\epsilon>0$ small so that $t_{0}<T-\epsilon$. From the formula of $w^{*}(t)$, clearly we can find some $c>0$ such that $w^{*}(t) \leq c t^{-\frac{1}{\underline{p}^{-1}}}$ for $t \in\left(0, t_{0}\right]$. On the other hand, we already have $z^{*}(x) \leq c_{2} d(x)^{-\frac{2+\beta}{p-1}}$ in $\Omega$. Hence (1.7) follows from $\bar{u} \leq M\left(w^{*}+z^{*}\right)$.

It remains to prove (1.6). Choose $M_{\epsilon}>0$ such that $\alpha_{2}(t) \leq M_{\epsilon}$ on $[0, T-\epsilon]$. We also note that $|a| \leq A$ on $\bar{\Omega} \times[0, T]$. Then, for any small $\delta>0$, we consider the following auxiliary problems:

$$
w^{\prime}=-A w-w^{\bar{p}_{\beta}} \quad \text { for } t>-\delta, \quad w(-\delta)=\infty,
$$

and

$$
-\Delta z=-A z-d(x)^{\beta} z^{p} \quad \text { in } \Omega, \quad z=\infty \text { on } \partial \Omega .
$$

The unique solution $w_{*}^{\delta}(t)$ of $(2.9)$ can be explicitly written as

$$
w_{*}^{\delta}(t)=w_{*}(t+\delta), w_{*}(t)=A^{\frac{1}{\bar{p}^{-1}}} e^{-A t}\left[1-e^{-A\left(\bar{p}_{\beta}-1\right) t}\right]^{\frac{1}{1-\bar{p} \beta}} .
$$

Clearly, $w_{*}^{\delta} \rightarrow w_{*}$ locally uniformly on $(0, T]$ as $\delta \rightarrow 0$, and $w_{*}(t)$ is the unique solution of

$$
w^{\prime}=-A w-w^{\bar{p}_{\beta}} \quad \text { for } t>0, \quad w(0)=\infty .
$$

On the other hand, similarly to (2.3), it is well-known that problem (2.10) possesses a unique positive solution $z_{*}(x)$, and

$$
\lim _{x \rightarrow \partial \Omega} \frac{z_{*}(x)}{d(x)^{-\frac{2+\beta}{p-1}}}=\left[\frac{(2+\beta)(1+\beta+p)}{(p-1)^{2}}\right]^{\frac{1}{p-1}} .
$$

Hence, there is a constant $c_{0}>1$ such that

$$
c_{0}^{-1} d(x)^{-\frac{2+\beta}{p-1}} \leq z_{*}(x) \leq c_{0} d(x)^{-\frac{2+\beta}{p-1}} \quad \text { in } \Omega .
$$

If $\beta \geq 0$, then $\bar{p}_{\beta}=p$ and $c_{6} d(x)^{\beta} \leq 1$ for some positive constant $c_{6}$. Thus we have

$$
\left(w_{*}^{\delta}\right)^{\prime}=-A w_{*}^{\delta}-\left(w_{*}^{\delta}\right)^{p} \leq-A w_{*}^{\delta}-c_{6} d(x)^{\beta}\left(w_{*}^{\delta}\right)^{p} .
$$

It follows that for $m>0$ small enough (independent of $\delta$ ), $U_{*}=m\left(w_{*}^{\delta}+z_{*}\right)$ satisfies

$$
\left(U_{*}\right)_{t}-\Delta U_{*} \leq-A U_{*}-M_{\epsilon} d(x)^{\beta} U_{*}^{p} \quad(x \in \Omega, t \geq-\delta) .
$$


Therefore,

$$
\left(U_{*}\right)_{t}-\Delta U_{*} \leq a(x, t) U_{*}-b(x, t) U_{*}^{p} \quad(x \in \Omega, \quad t \in[0, T-\epsilon]) .
$$

If $\beta \in(-2,0)$, then

$$
\underline{p}_{\beta}=p-\beta \frac{p-1}{2+\beta}>p
$$

and there exists $c_{7}>0$ such that $d(x)^{\beta} \geq c_{7} z_{*}(x)^{\bar{p}_{\beta}-p}$. Hence

$$
\left(z_{*}\right)_{t}-\Delta z_{*} \leq-A z_{*}-c_{7}\left(z_{*}\right)^{\bar{p}_{\beta}} .
$$

Therefore, for $m_{1}>0$ small, $U_{*}^{1}:=m_{1}\left(w_{*}^{\delta}+z_{*}\right)$ satisfies

$$
\left(U_{*}^{1}\right)_{t}-\Delta U_{*}^{1} \leq-A U_{*}^{1}-\left(U_{*}^{1}\right)^{\bar{p}_{\beta}} \quad(x \in \Omega, t>-\delta) .
$$

Clearly there exists some $c_{8}>0$ such that

$$
\left(U_{*}^{1}\right)^{\bar{p}_{\beta}-p} \geq\left(m_{1} z_{*}\right)^{\bar{p}_{\beta}-p} \geq c_{8} d(x)^{\beta} \quad(x \in \Omega) .
$$

Therefore

$$
\left(U_{*}^{1}\right)_{t}-\Delta U_{*}^{1} \leq-A U_{*}^{1}-c_{8} d(x)^{\beta}\left(U_{*}^{1}\right)^{p} \quad(x \in \Omega, t>-\delta) .
$$

This implies that for all small $m \in\left(0, m_{1}\right), U_{*}:=m\left(w_{*}^{\delta}+z_{*}\right)$ satisfies

$$
\left(U_{*}\right)_{t}-\Delta U_{*} \leq-A U_{*}-M_{\epsilon} d(x)^{\beta}\left(U_{*}\right)^{p} \quad(x \in \Omega, t>-\delta),
$$

and hence (2.12) holds.

By a standard argument (see, for example, the proof of Theorem 6.14 in [6]), the problem

$$
-\Delta z=-A z-d(x)^{\beta} z^{p} \text { in } \Omega, z=n \text { on } \partial \Omega
$$

has a unique solution $z_{n}$, and $z_{n} \rightarrow z_{*}$ locally uniformly in $\Omega$ as $n \rightarrow \infty$. Moreover, after further shrinking $m$ if needed, it is easily seen by the comparison principle that $\underline{u}(x, t) \geq 2 m z_{n}(x)$ in $\Omega \times(0, T-\epsilon]$ for all $n \geq 1$. It follows that $\underline{u}(x, t) \geq 2 m z_{*}(x)$ in $\Omega \times(0, T-\epsilon]$. Thus

$$
\liminf _{(x, t) \rightarrow \Sigma_{\epsilon}}\left[\underline{u}(x, t)-U_{*}(x, t)\right] \geq 0,
$$

where $\Sigma_{\epsilon}=(\partial \Omega \times(0, T-\epsilon]) \cup(\Omega \times\{0\})$. In view of (2.12), we may now apply the maximum principle to deduce that

$$
\underline{u}(x, t) \geq U_{*}(x, t)=m\left(w_{*}^{\delta}(t)+z_{*}(x)\right) \text { in } \Omega \times(0, T-\epsilon] .
$$

Letting $\delta \rightarrow 0$ we obtain

$$
\underline{u}(x, t) \geq m\left(w_{*}(t)+z_{*}(x)\right) \text { in } \Omega \times(0, T-\epsilon] .
$$

The inequality (1.6) now follows easily from the behavior of $w_{*}(t)$ and $z_{*}(x)$. The proof is complete.

To show that under the assumption $c(T-t)^{\theta} d(x)^{\beta} \leq b(x, t)$ on $\Omega \times[0, T)$, the maximal solution of (1.1) satisfies (1.8), by a simple comparison argument, it is sufficient to establish the following result. 
Theorem 2.1. Assume that $a, \beta, \theta$ are constants with $a>0, \theta>0, \beta>-2$, and $\bar{u}(x, t)$ is the maximal solution to

$$
\begin{cases}u_{t}-\Delta u=a u-(T-t)^{\theta} d(x)^{\beta} u^{p} & \text { in } \Omega \times(0, T), \\ u=\infty & \text { on } \partial \Omega \times(0, T) \cup \bar{\Omega} \times\{0\} .\end{cases}
$$

Then (1.8) holds.

Proof. Let

$$
\bar{u}(x, t)=(T-t)^{-\frac{\theta}{p-1}} e^{a t} v(x, t)
$$

A simple calculation shows that $v(x, t)$ satisfies

$$
\begin{cases}v_{t}-\Delta v=-\frac{\theta}{p-1}(T-t)^{-1} v-e^{a(p-1) t} d(x)^{\beta} v^{p} & \text { in } \Omega \times(0, T), \\ v=\infty & \text { on } \partial \Omega \times(0, T), \\ v=\infty & \text { on } \bar{\Omega} \times\{0\} .\end{cases}
$$

Let $z_{0}(x)$ be the unique positive solution of

$$
-\Delta z=-d(x)^{\beta} z^{p} \text { in } \Omega, z=\infty \text { on } \partial \Omega .
$$

For any given $t_{0} \in(0, T)$, due to (1.7) (applied to $v(x, t)$ ) and the behavior of $z_{0}(x)$ near $\partial \Omega(\mathrm{cp} .(2.4))$, there exists a constant $c_{1} \geq 1$ such that

$$
v\left(t_{0}, x\right) \leq c_{1} z_{0}(x) \text { in } \Omega .
$$

Let $z_{\sigma}$ be the unique positive solution of (2.16) with $\Omega$ replaced by $\Omega_{\sigma}:=\{x \in \Omega: d(x)>$ $\sigma\}$, with $\sigma>0$ small. Then $z_{\sigma}>z_{0}$ in $\Omega_{\sigma}$ and $z_{\sigma} \rightarrow z_{0}$ as $\sigma \rightarrow 0$. A simple comparison consideration shows that $v(x, t) \leq c_{1} z_{\sigma}(x)$ in $\Omega_{\sigma} \times\left[t_{0}, T\right)$. Letting $\sigma \rightarrow 0$ we deduce

$$
v(x, t) \leq c_{1} z_{0}(x) \text { in } \Omega \times\left[t_{0}, T\right) .
$$

Since

$$
z_{0}(x) \leq c_{2} d(x)^{-\frac{2+\beta}{p-1}} \text { in } \Omega
$$

for some $c_{2}>0$, we thus obtain, for some $c_{3}>0$,

$$
\bar{u}(x, t) \leq c_{3}(T-t)^{-\frac{\theta}{p-1}} d(x)^{-\frac{2+\beta}{p-1}} \text { in } \Omega \times\left[t_{0}, T\right) .
$$

Define

$$
M(x, t):=\bar{u}(x, t) d(x)^{\frac{2+2 \theta+\beta}{p-1}} \text { for }(x, t) \in \Omega \times\left[t_{0}, T\right),
$$

and

$$
M(t):=\sup _{x \in \Omega} M(x, t) \text { for } t \in\left[t_{0}, T\right) .
$$

We prove that there exists $C>0$ such that

$$
M(t) \leq C \quad\left(t \in\left[t_{0}, T\right)\right)
$$

Clearly, (1.8) is a consequence of (2.17) and (2.18). 
Thus to prove the theorem, it suffices to show (2.18). We argue indirectly. Suppose that (2.18) does not hold. Then we can find a sequence $t_{n}$ increasing to $T$ as $n \rightarrow \infty$, such that

$$
M\left(t_{n}\right) \rightarrow \infty, M(t) \leq M\left(t_{n}\right) \quad\left(t \in\left[t_{0}, t_{n}\right]\right) .
$$

From (2.17) we see that $M(x, t)=0$ for $x \in \partial \Omega$. Thus there exists $x_{n} \in \Omega$ such that

$$
M_{n}:=M\left(t_{n}\right)=M\left(x_{n}, t_{n}\right) .
$$

We now define

$$
\lambda_{n}=\bar{u}\left(x_{n}, t_{n}\right)^{-\frac{1}{\sigma}} \text { with } \sigma=\frac{2 \theta+2}{p-1},
$$

and

$$
v_{n}(y, s)=\lambda_{n}^{\sigma} \bar{u}\left(x_{n}+\lambda_{n} d\left(x_{n}\right)^{\xi} y, t_{n}+\lambda_{n}^{2} d\left(x_{n}\right)^{2 \xi} s\right) \text { for }(y, s) \in D_{n},
$$

where $\xi=-\beta /(2+2 \theta)$ and

$$
D_{n}:=\left\{(y, s): \underline{s}_{n} \leq s \leq 0, x_{n}+\lambda_{n} d\left(x_{n}\right)^{\xi} y \in \Omega\right\}, \underline{s}_{n}:=-\frac{t_{n}-t_{0}}{\lambda_{n}^{2} d\left(x_{n}\right)^{2 \xi}} .
$$

A direct calculation yields

$$
\begin{aligned}
\partial_{s} v_{n} & -\Delta_{y} v_{n}=\lambda_{n}^{\sigma+2} d\left(x_{n}\right)^{2 \xi}\left(\bar{u}_{t}-\Delta \bar{u}\right) \\
& =\lambda_{n}^{2} d\left(x_{n}\right)^{2 \xi} a v_{n}-\lambda_{n}^{2+\sigma-\sigma p+2 \theta} d\left(x_{n}\right)^{2 \xi+\beta+2 \theta \xi}\left(\eta_{n}-s\right)^{\theta}\left(\frac{d\left(x_{n}+\lambda_{n} d\left(x_{n}\right)^{\xi} y\right)}{d\left(x_{n}\right)}\right)^{\beta} v_{n}^{p},
\end{aligned}
$$

with

$$
\eta_{n}=\frac{T-t_{n}}{\lambda_{n}^{2} d\left(x_{n}\right)^{2 \xi}}>0 .
$$

By the choice of $\sigma$ and $\xi$, we have

$$
2+\sigma-\sigma p+2 \theta=0,2 \xi+\beta+2 \theta \xi=0 .
$$

Thus

$$
\partial_{s} v_{n}-\Delta_{y} v_{n}=\lambda_{n}^{2} d\left(x_{n}\right)^{2 \xi} a v_{n}-\left(\eta_{n}-s\right)^{\theta}\left(\frac{d\left(x_{n}+\lambda_{n} d\left(x_{n}\right)^{\xi} y\right)}{d\left(x_{n}\right)}\right)^{\beta} v_{n}^{p} \quad\left((y, s) \in D_{n}\right)
$$

Moreover, by the definitions of $t_{n}, x_{n}$ and $\lambda_{n}$, we have $v_{n}(0,0)=1$ and

$$
\begin{aligned}
\lambda_{n} d\left(x_{n}\right)^{\xi} & =\bar{u}\left(x_{n}, t_{n}\right)^{-1 / \sigma} d\left(x_{n}\right)^{\xi} \\
& =M_{n}^{-1 / \sigma} d\left(x_{n}\right) \rightarrow 0 \text { as } n \rightarrow \infty .
\end{aligned}
$$

It follows that

$$
\underline{s}_{n} \rightarrow-\infty, \quad R_{n}:=\frac{d\left(x_{n}\right)}{2 \lambda_{n} d\left(x_{n}\right)^{\xi}}=M_{n}^{1 / \sigma} / 2 \rightarrow \infty \text { as } n \rightarrow \infty .
$$

Define

$$
D_{n}^{0}:=\left\{(y, s): \underline{s}_{n} \leq s \leq 0,|y| \leq R_{n}\right\}
$$


Then from the definition of $R_{n}$ we find that $D_{n}^{0} \subset D_{n}$ for all $n \geq 1$. Furthermore, for $(y, s) \in D_{n}^{0}$,

$$
\frac{d\left(x_{n}+\lambda_{n} d\left(x_{n}\right)^{\xi} y\right)}{d\left(x_{n}\right)} \leq \frac{d\left(x_{n}\right)+\lambda_{n} d\left(x_{n}\right)^{\xi}|y|}{d\left(x_{n}\right)} \leq \frac{d\left(x_{n}\right)+\lambda_{n} d\left(x_{n}\right)^{\xi} R_{n}}{d\left(x_{n}\right)}=\frac{3}{2},
$$

and

$$
\frac{d\left(x_{n}+\lambda_{n} d\left(x_{n}\right)^{\xi} y\right)}{d\left(x_{n}\right)} \geq \frac{d\left(x_{n}\right)-\lambda_{n} d\left(x_{n}\right)^{\xi}|y|}{d\left(x_{n}\right)} \geq \frac{d\left(x_{n}\right)-\lambda_{n} d\left(x_{n}\right)^{\xi} R_{n}}{d\left(x_{n}\right)}=\frac{1}{2} .
$$

Consequently,

$$
\left(\frac{d\left(x_{n}+\lambda_{n} d\left(x_{n}\right)^{\xi} y\right)}{d\left(x_{n}\right)}\right)^{\beta} \geq c_{1}:=\min \left\{(1 / 2)^{\beta},(3 / 2)^{\beta}\right\} .
$$

From the definition of $\lambda_{n}$ and (2.20), we also obtain

$$
\begin{aligned}
0 \leq v_{n}(y, s) & =\lambda_{n}^{\sigma} M\left(x_{n}+\lambda_{n} d\left(x_{n}\right)^{\xi} y, t_{n}+\lambda_{n}^{2} d\left(x_{n}\right)^{2 \xi} s\right) d\left(x_{n}+\lambda_{n} d\left(x_{n}\right)^{\xi} y\right)^{-\frac{2+2 \theta+\beta}{p-1}} \\
& \leq \lambda_{n}^{\sigma} \bar{u}\left(x_{n}, t_{n}\right)\left[\frac{d\left(x_{n}+\lambda_{n} d\left(x_{n}\right)^{\xi} y\right)}{d\left(x_{n}\right)}\right]^{-\frac{2+2 \theta+\beta}{p-1}} \\
& \leq C_{0}:=2^{\frac{2+2 \theta+\beta}{p-1}} .
\end{aligned}
$$

Denoting $\epsilon_{n}:=\lambda_{n}^{2} d\left(x_{n}\right)^{2 \xi} a$, we have $\epsilon_{n} \rightarrow 0$ as $n \rightarrow \infty$, and

$$
\partial_{s} v_{n}-\Delta_{y} v_{n} \leq \epsilon_{n} v_{n}-c_{1}(-s)^{\theta} v_{n}^{p}, 0 \leq v_{n} \leq C_{0} \text { in } D_{n}^{0}, v_{n}(0,0)=1
$$

for all $n \geq 1$.

We now fix $\delta \in(0,1)$ and consider, for $\epsilon>0$ to be determined later, the ODE problem

$$
w^{\prime}=\epsilon w-c_{1}(-s)^{\theta} w^{p} \text { for } s \leq 0, w(0)=\delta .
$$

The unique solution of (2.22) satisfies, for $s<0, w(s)=e^{\epsilon s} w_{0}(s)$, and

$$
\begin{aligned}
w_{0}(s)^{1-p} & =\delta^{1-p}-\int_{s}^{0} c_{1}(p-1)(-t)^{\theta} e^{\epsilon(p-1) t} d t \\
& =\delta^{1-p}-\frac{c_{1}(p-1)}{[\epsilon(p-1)]^{1+\theta}} \int_{\epsilon(p-1) s}^{0}(-t)^{\theta} e^{t} d t .
\end{aligned}
$$

Set

$$
C^{0}:=\int_{-\infty}^{0}(-t)^{\theta} e^{t} d t
$$

and fix $\epsilon>0$ small enough such that

$$
\delta^{1-p}<\frac{c_{1}(p-1)}{[\epsilon(p-1)]^{1+\theta}} C^{0} .
$$

Then there exists a unique $s_{0}<0$ such that $w_{0}(s) \rightarrow+\infty$ as $s$ decreases to $s_{0}$, and $w_{0}(s)>0$ in $\left(s_{0}, 0\right]$. Hence $w(s) \rightarrow+\infty$ as $s$ decreases to $s_{0}$, and $w(s)>0$ in $\left(s_{0}, 0\right]$.

For the above chosen $\epsilon>0$ we define $Z(y)=\frac{\epsilon}{4 N}|y|^{2}+1$. Clearly

$$
-\Delta Z=-\frac{\epsilon}{2} \geq-\frac{\epsilon}{2} Z \quad\left(y \in \mathbb{R}^{N}\right) .
$$


It follows that, for $V(y, s)=w(s) Z(y)$ and $(y, s) \in \mathbb{R}^{N} \times\left(s_{0}, 0\right]$,

$$
\begin{aligned}
V_{s}-\Delta V & =w^{\prime}(s) Z(y)-w(s) \Delta Z(y) \\
& \geq\left[\epsilon w-c_{1}(-s)^{\theta} w^{p}\right] Z-\frac{\epsilon}{2} w Z \\
& =\frac{\epsilon}{2} w Z-c_{1}(-s)^{\theta} w^{p} Z \\
& \geq \frac{\epsilon}{2} V-c_{1}(-s)^{\theta} V^{p} .
\end{aligned}
$$

Clearly $V(y, s) \rightarrow+\infty$ uniformly in $y$ as $s$ decreases to $s_{0}$, and

$$
V(y, s) \geq \min _{s \in\left(s_{0}, 0\right]} w(s)\left(\frac{\epsilon}{4 N}|y|^{2}+1\right) \geq C_{0} \text { for }|y|=R_{n} \text { and all large } n \text {. }
$$

Moreover, $\epsilon / 2 \geq \epsilon_{n}$ for all large $n$. Thus in view of (2.21), we can apply the comparison principle to conclude that

$$
v_{n}(y, s) \leq V(y, s) \text { for }|y| \leq R_{n}, s \in\left(s_{0}, 0\right] \text { and all large } n .
$$

In particular,

$$
1=v_{n}(0,0) \leq V(0,0)=\delta .
$$

This contradiction completes the proof.

\section{LOCAL BEHAVIOR AT THE PARABOLIC BOUNDARY AND UNIQUENESS}

Throughout this section, we assume that (1.4) and (1.5) hold, and $u(x, t)$ is an arbitrary positive solution of (1.1).

We first consider the behavior of $u(x, t)$ near $\partial \Omega \times(0, T)$.

Theorem 3.1. Let $u$ be a positive solution of (1.1). Suppose that $\gamma(x, t):=b(x, t) / d(x)^{\beta}$ extends to a continuos function on $\bar{\Omega} \times(0, T)$. Then, for $x_{0} \in \partial \Omega$ and $t_{0} \in(0, T)$,

$$
\lim _{x \rightarrow x_{0}, x \in \Omega} \frac{u\left(x, t_{0}\right)}{d(x)^{-\frac{2+\beta}{p-1}}}=\left[\frac{(2+\beta)(p+\beta+1)}{\gamma\left(x_{0}, t_{0}\right)(p-1)^{2}}\right]^{\frac{1}{p-1}} .
$$

Proof. Fix $x_{0} \in \partial \Omega$ and $t_{0} \in(0, T)$ and let

$$
a_{0}:=a\left(x_{0}, t_{0}\right), \gamma_{0}:=\gamma\left(x_{0}, t_{0}\right) .
$$

For any given small $\epsilon \in\left(0, \gamma_{0} / 2\right)$, we can find $\delta \in\left(0, t_{0}\right)$ small enough such that, for $(x, t) \in \Omega \times(0, T)$ satisfying $\left|x-x_{0}\right|<2 \delta$ and $\left|t-t_{0}\right|<\delta$, the following holds:

$$
a_{0}-\epsilon \leq a(x, t) \leq a_{0}+\epsilon, \gamma_{0}-\epsilon \leq \frac{b(x, t)}{d(x)^{\beta}} \leq \gamma_{0}+\epsilon .
$$

Let $B^{0} \subset \Omega \cap B_{2 \delta}\left(x_{0}\right)$ be a smooth domain such that $\partial B^{0}$ and $\partial \Omega$ coincide inside $B_{\delta}\left(x_{0}\right)$, and let $v(x)$ be a positive solution of

$$
-\Delta v=\left(a_{0}+\epsilon\right) v-\left(\gamma_{0}-2 \epsilon\right) d(x)^{\beta} v^{p} \quad \text { in } B^{0}, \quad v=\infty \quad \text { on } \partial B^{0} .
$$


Then we have by [6] and [4],

$$
\lim _{x \rightarrow x_{0}} \frac{v(x)}{d(x)^{-\frac{2+\beta}{p-1}}}=C_{0}:=\left[\frac{(2+\beta)(1+\beta+p)}{\left(\gamma_{0}-2 \epsilon\right)(p-1)^{2}}\right]^{\frac{1}{p-1}} .
$$

In the case $\beta \geq 0$, the above limit follows from Theorem 2.5 of $[6]$, and the case $\beta \in(-2,0)$ can be proved by the same argument as in section 4 of [4].

Thus we can find $\delta_{1} \in(0, \delta)$ such that

$$
v(x)^{1-p} \leq 2 C_{0}^{1-p} d(x)^{2+\beta} \leq \epsilon \delta(p-1) d(x)^{\beta} \quad\left(x \in B^{0} \cap B_{2 \delta_{1}}\left(x_{0}\right)\right) .
$$

We now consider $\Omega_{\sigma}:=\{x \in \Omega: d(x)>\sigma\}$ for sufficiently small $\sigma \in[0, \delta)$. For each such $\Omega_{\sigma}$ we can construct a smooth domain $B_{\sigma}^{0} \subset \Omega_{\sigma} \cap B_{2 \delta_{1}}\left(x_{0}\right) \subset B^{0}$ such that $\partial B_{\sigma}^{0}$ and $\partial \Omega_{\sigma}$ coincide inside $B_{\delta_{1}}\left(x_{0}\right)$, and $B_{\sigma}^{0}$ varies continuously with $\sigma$ for all small nonnegative $\sigma$. We may also require that $B_{\sigma}^{0} \subset B_{\sigma^{\prime}}^{0}$ when $\sigma>\sigma^{\prime}$. Let $v_{\sigma}$ be the maximal positive solution of

$$
-\Delta v=\left(a_{0}+\epsilon\right) v-\left(\gamma_{0}-2 \epsilon\right) d(x)^{\beta} v^{p} \quad \text { in } B_{\sigma}^{0}, \quad v=\infty \quad \text { on } \partial B_{\sigma}^{0} .
$$

A standard comparison argument (see, for example, Proposition 2.1 in [6]) shows that $v_{\sigma} \geq v$ in $B_{\sigma}^{0}$, and by further using the elliptic regularity and the maximality of $v_{0}$, we see that $v_{\sigma}$ decreases to $v_{0}$ as $\sigma$ decreases to 0 . Therefore

$$
v_{\sigma}(x)^{1-p} \leq v(x)^{1-p} \leq \epsilon \sigma(p-1) d(x)^{\beta} \quad\left(x \in B_{\sigma}^{0}\right) .
$$

Define

$$
\eta(t)=\left[1+\delta^{-1}\left(t-t_{0}\right)\right]^{1 /(1-p)} \text { for } t \in\left(t_{0}-\delta, t_{0}\right] .
$$

It is easily checked that

$$
\eta^{\prime}=-\frac{1}{\delta(p-1)} \eta^{p}, \eta \geq 1 \text { in }\left(t_{0}-\delta, t_{0}\right], \eta\left(t_{0}-\delta\right)=+\infty, \eta\left(t_{0}\right)=1 .
$$

Set

$$
u_{\sigma}(x, t)=\eta(t) v_{\sigma}(x) .
$$

Then, due to (3.1), for $(x, t) \in B_{\sigma}^{0} \times\left(t_{0}-\delta, t_{0}\right]$, we have

$$
\begin{aligned}
\left(u_{\sigma}\right)_{t}-\Delta u_{\sigma} & =\eta^{\prime}(t) v_{\sigma}(x)-\eta(t) \Delta v_{\sigma}(x) \\
& =\left(a_{0}+\epsilon\right) u_{\sigma}-\frac{1}{\delta(p-1)} v_{\sigma}^{1-p} u_{\sigma}^{p}-\left(\gamma_{0}-2 \epsilon\right) d(x)^{\beta} \eta(t)^{1-p} u_{\sigma}^{p} \\
& \geq\left(a_{0}+\epsilon\right) u_{\sigma}-\left(\gamma_{0}-\epsilon\right) d(x)^{\beta} u_{\sigma}^{p} .
\end{aligned}
$$

It follows from the comparison principle that

$$
u(x, t) \leq u_{\sigma}(x, t)=\eta(t) v_{\sigma}(x) \quad\left((x, t) \in B_{\sigma}^{0} \times\left(t_{0}-\delta, t_{0}\right]\right) .
$$

Letting $\sigma \rightarrow 0$, we deduce

$$
u(x, t) \leq \eta(t) v_{0}(x) \quad\left((x, t) \in B_{0}^{0} \times\left(t_{0}-\delta, t_{0}\right]\right) .
$$


Hence

$$
\limsup _{x \rightarrow x_{0}, x \in \Omega} \frac{u\left(x, t_{0}\right)}{d(x)^{-\frac{2+\beta}{p-1}}} \leq \lim _{x \rightarrow x_{0}, x \in B_{0}^{0}} \frac{v_{0}(x)}{d(x)^{-\frac{2+\beta}{p-1}}}=\left[\frac{(2+\beta)(1+\beta+p)}{\left(\gamma_{0}-2 \epsilon\right)(p-1)^{2}}\right]^{\frac{1}{p-1}}
$$

where we have applied Theorem 2.5 of [6] and the arguments in section 4 of [4] to $v_{0}(x)$.

We show next that

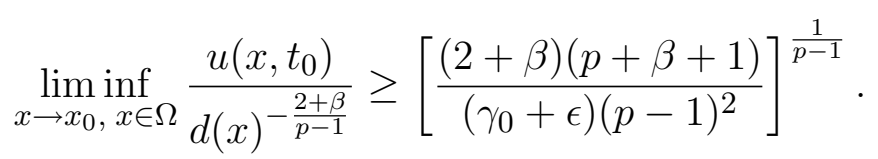

Let $w^{\sigma}$ be the unique positive solution of

$$
-\Delta w=\left(a_{0}-\epsilon\right) w-\left(\gamma_{0}+\epsilon\right) d(x)^{\beta} w^{p} \quad \text { in } \Omega_{\sigma},\left.w\right|_{\partial \Omega_{\sigma}}=\infty .
$$

Then from the comparison principle we find that $w^{\sigma}$ decreases to some $w^{0}$ as $\sigma$ decreases to 0 , and by elliptic regularity we see that $w^{0}$ is a positive solution of (3.4) with $\sigma=0$.

By the asymptotic behavior of $w^{0}$ and $u\left(x, t_{0}-\delta\right)$ near $\partial \Omega(\mathrm{cp}$. (1.6)), we can find $\epsilon_{0} \in(0,1)$ sufficiently small so that

$$
\epsilon_{0} w^{0}(x) \leq u\left(x, t_{0}-\delta\right) \text { in } \Omega .
$$

With $\epsilon_{0}>0$ fixed as above, we let $\alpha(t)$ be a smooth increasing function on $\left[t_{0}-\delta, t_{0}\right]$ with $\alpha\left(t_{0}-\delta\right)=\epsilon_{0}, \alpha\left(t_{0}\right)=1$, and define

$$
L:=\max _{\left[t_{0}-\delta, t_{0}\right]} \frac{\alpha^{\prime}(t)}{\alpha(t)} .
$$

We then choose $B_{2 \delta}\left(x_{0}\right)$ as before, set $B_{0}:=\Omega \cap B_{\delta}\left(x_{0}\right)$, and consider the auxiliary problem

$$
-\Delta w=\left(a_{0}-\epsilon-L\right) w-\left(\gamma_{0}+\epsilon\right) d(x)^{\beta} w^{p} \quad \text { in } B_{0},\left.w\right|_{\partial B_{0} \cap \partial \Omega}=\infty,\left.w\right|_{\partial B_{0} \cap \Omega}=0 .
$$

If $\beta \geq 0$ then we can apply Theorem 1.1 of [7] to conclude that (3.5) has a positive solution $w_{0}$. In what follows we use a variant of the argument in [7] to show that this is true for the entire range $\beta>-2$.

Let $\phi_{n}$ be smooth functions over $\bar{\Omega}$ such that $\left.\phi_{n}\right|_{\partial B_{0} \cap \Omega}=0$ and $\phi_{n}$ increases to $\infty$ as $n \rightarrow \infty$ on any compact subset of $\partial B_{0} \cap \partial \Omega$. Then for each $m \geq 1$ the problem

$$
-\Delta v=\left(a_{0}-\epsilon-L\right) w-\left(\gamma_{0}+\epsilon\right) \min \left\{d(x)^{\beta}, m\right\} v^{p} \text { in } B_{0},\left.v\right|_{\partial B_{0}}=\phi_{n}
$$

has a unique positive solution $v^{m}$. By the comparison principle we have $v^{m} \geq v^{m+1}$. Thus $v=\lim _{m \rightarrow \infty} v^{m}$ exists, and one easily sees by standard elliptic regularity that $v$ is a solution to

$$
-\Delta v=\left(a_{0}-\epsilon-L\right) w-\left(\gamma_{0}+\epsilon\right) d(x)^{\beta} v^{p} \text { in } B_{0},\left.v\right|_{\partial B_{0}}=\phi_{n} .
$$

Using Proposition 2.1 of [6], we conclude that such a solution $v$ is unique, and we may denote it by $v_{n}$. Moreover, this comparison principle also infers that $v_{n} \leq v_{n+1} \leq w^{\sigma}$ in $B_{0} \cap \Omega_{\sigma}$. Hence $v_{*}:=\lim _{n \rightarrow \infty} v_{n}$ exists, and by elliptic regularity we find that $v_{*}$ is 
a positive solution of (3.5). Moreover $v_{*} \leq w^{\sigma}$ in $B_{0} \cap \Omega_{\sigma}$. Letting $\sigma \rightarrow 0$ we deduce $v_{*} \leq w^{0}$ in $B_{0}$.

Set $u^{n}(x, t)=\alpha(t) v_{n}(x)$ for $(x, t) \in B_{0} \times\left[t_{0}-\delta, t_{0}\right]$. Clearly

$$
u^{n}\left(x, t_{0}-\delta\right)=\epsilon_{0} v_{n}(x) \leq \epsilon_{0} v_{*}(x) \leq \epsilon_{0} w^{0}(x) \leq u\left(x, t_{0}-\delta\right) \text { for } x \in B_{0} .
$$

It is also evident that $u^{n} \leq u$ on $\partial B_{0} \times\left[t_{0}-\delta, t_{0}\right]$. Moreover, in $B_{0} \times\left[t_{0}-\delta, t_{0}\right]$,

$$
\begin{aligned}
u_{t}^{n}-\Delta u^{n} & =\left(\frac{\alpha^{\prime}(t)}{\alpha(t)}+a_{0}-\epsilon-L\right) u^{n}-\left(\gamma_{0}+\epsilon\right) d(x)^{\beta}\left(u^{n}\right)^{p} \alpha(t)^{1-p} \\
& \leq\left(a_{0}-\epsilon\right) u^{n}-\left(\gamma_{0}+\epsilon\right) d(x)^{\beta}\left(u^{n}\right)^{p} .
\end{aligned}
$$

Hence we can apply the comparison principle to deduce that

$$
u^{n}(x, t) \leq u(x, t) \text { in } B_{0} \times\left[t_{0}-\delta, t_{0}\right] .
$$

In particular,

$$
v_{n}(x) \leq u\left(x, t_{0}\right) \text { in } B_{0} .
$$

Letting $n \rightarrow \infty$ we deduce $v_{*}(x) \leq u\left(x, t_{0}\right)$ in $B_{0}$. It follows that

$$
\liminf _{x \rightarrow x_{0}, x \in \Omega} \frac{u\left(x, t_{0}\right)}{d(x)^{-\frac{2+\beta}{p-1}}} \geq \lim _{x \rightarrow x_{0}, x \in B_{0}} \frac{v_{*}(x)}{d(x)^{-\frac{2+\beta}{p-1}}}=\left[\frac{(2+\beta)(p+\beta+1)}{\left(\gamma_{0}+\epsilon\right)(p-1)^{2}}\right]^{\frac{1}{p-1}},
$$

where, as before, we have used Theorem 2.5 of [6] and section 4 of [4] to obtain the asymptotic behavior of $v_{*}$. Hence (3.3) holds. The desired result clearly follows from (3.2) and (3.3), since $\epsilon>0$ can be arbitrarily small.

Next, we study the blow-up rate of the solution at the initial time $t=0$.

Theorem 3.2. Let $u$ be a positive solution of (1.1). Then,

$$
\lim _{t \rightarrow 0} t^{\frac{1}{p-1}} u\left(x_{0}, t\right)=\left[(p-1) b\left(x_{0}, 0\right)\right]^{\frac{1}{1-p}},\left(x_{0} \in \Omega\right) .
$$

Proof. We take a fixed $x_{0} \in \Omega$. Then, for any given small $\epsilon>0$, we can find a small ball $B_{R}\left(x_{0}\right)$ and small $t_{0}>0$ such that $\bar{B}_{R}\left(x_{0}\right) \subset \Omega$ and

$$
a_{0}-\epsilon \leq a(x, t) \leq a_{0}+\epsilon, 0<b_{0}-\epsilon \leq b(x, t) \leq b_{0}+\epsilon
$$

for all $x \in B_{R}\left(x_{0}\right), t \in\left[0, t_{0}\right]$, where $a_{0}:=a\left(x_{0}, 0\right)$ and $b_{0}:=b\left(x_{0}, 0\right)$.

We show that

$$
\limsup _{t \rightarrow 0}\left[(p-1)\left(\left(b_{0}-\epsilon\right)\right) t\right]^{\frac{1}{p-1}} u\left(x_{0}, t\right) \leq 1 .
$$

This follows from a localization argument based on the results of [3]. But for completeness we give a simple alternative proof. Let $\tilde{w}$ and $\tilde{z}$ be the solution of the following problems, respectively:

$$
w^{\prime}=\left(a_{0}+\epsilon\right) w-\left(b_{0}-\epsilon\right) w^{p}, \quad t>0 ; \quad w(0)=\infty,
$$

and

$$
-\Delta z=\left(a_{0}+\epsilon\right) z-\left(b_{0}-\epsilon\right) z^{p} \quad \text { in } B_{R}\left(x_{0}\right), \quad z=\infty \text { on } \partial B_{R}\left(x_{0}\right)
$$


By a simple comparison argument, we have

$$
u \leq \tilde{w}+\tilde{z} \text { on } B_{R}\left(x_{0}\right) \times\left(0, t_{0}\right] .
$$

From the explicit expression of $\tilde{w}(t)$ we easily find

$$
\left[(p-1)\left(b_{0}-\epsilon\right) t\right]^{\frac{1}{p-1}} \tilde{w}(t) \rightarrow 1 \text { as } t \rightarrow 0 .
$$

Furthermore, $\tilde{z}$ is bounded on $\bar{B}_{R / 2}\left(x_{0}\right)$. These facts, together with (3.7), enable us to obtain (3.6).

Next we prove that

$$
\liminf _{t \rightarrow 0}\left[(p-1)\left(b_{0}+\epsilon\right) t\right]^{\frac{1}{p-1}} u\left(x_{0}, t\right) \geq 1 .
$$

For this purpose, we let $\lambda_{1}$ be the first eigenvalue of the problem

$$
-\Delta \phi=\lambda \phi \quad \text { in } B_{R}\left(x_{0}\right), \quad \phi=0 \text { on } \partial B_{R}\left(x_{0}\right),
$$

and $\phi$ with $\sup _{B_{R}\left(x_{0}\right)} \phi=1$ be the positive eigenfunction corresponding to $\lambda_{1}$. Obviously, $0<\phi(x)<1$ in $B_{R}\left(x_{0}\right) \backslash\left\{x_{0}\right\}$ and $\phi\left(x_{0}\right)=1$.

Let $\underset{\sim}{w}$ denote the solution of

$$
w^{\prime}=\left[\left(a_{0}-\epsilon\right)-\lambda_{1}\right] w-\left(b_{0}+\epsilon\right) w^{p}, \quad t>0 ; \quad w(0)=\infty .
$$

Then, one easily checks that, for any small $\delta>0, \underset{\sim}{w}(t+\delta) \phi(x)$ is a subsolution to the following problem:

$$
\begin{cases}v_{t}-\Delta v=a(x, t) v-b(x, t) v^{p} & \text { in } B_{R}\left(x_{0}\right) \times\left(0, t_{0}\right), \\ v=u & \text { on } \partial B_{R}\left(x_{0}\right) \times\left(0, t_{0}\right) \cup \bar{B}_{R}\left(x_{0}\right) \times\{0\} .\end{cases}
$$

Clearly $u$ solves (3.9). The comparison argument then implies $\underset{\sim}{w}(t+\delta) \phi(x) \leq u(x, t)$ in $B_{R}\left(x_{0}\right) \times\left(0, t_{0}\right]$. Letting $\delta \rightarrow 0$ we deduce $\underset{\sim}{w}(t) \phi(x) \leq u(x, t)$ in $B_{R}\left(x_{0}\right) \times\left(0, t_{0}\right]$. In particular,

$$
\underset{\sim}{w}(t) \leq u\left(x_{0}, t\right) \quad\left(t \in\left(0, t_{0}\right]\right) .
$$

By the explicit expression of $\underset{\sim}{w}$, we have

$$
\left[(p-1)\left(\left(b_{0}+\epsilon\right)\right) t\right]^{\frac{1}{p-1}} \underset{\sim}{w}(t) \rightarrow 1 \text { as } t \rightarrow 0 .
$$

Thus it follows from (3.10) that (3.8) holds.

Combining (3.6) and (3.8), and taking into account that $\epsilon>0$ can be arbitrarily small, we deduce

$$
\left[(p-1) b\left(x_{0}, 0\right) t\right]^{\frac{1}{p-1}} u\left(x_{0}, t\right) \rightarrow 1 \quad \text { as } t \rightarrow 0,
$$

which is the desired result.

Finally, we use the convex function technique introduced by Marcus and Véron $[9,10]$ to show the uniqueness of positive solutions of (1.1) for the case $\beta=0$. 
Proof of Theorem 1.4: Clearly, it suffices to prove that $\underline{u}=\bar{u}$ in $\Omega \times(0, T-\epsilon)$ for any fixed $0<\epsilon<T$. Since $\bar{p}_{\beta}=\underline{p}_{\beta}$ when $\beta=0$, from (1.6) and (1.7) we find that when $\beta=0$, there exists a constant $k>1$ such that

$$
\underline{u} \leq \bar{u} \leq k \underline{u} \quad \text { in } \Omega \times(0, T-\epsilon) .
$$

To prove $\underline{u} \equiv \bar{u}$, we argue by contradiction. Assume that $\underline{u} \leq, \not \equiv \bar{u}$ in $\Omega \times(0, T-\epsilon)$. Then, by the strong maximum principle for parabolic equations, it is easily seen that $\underline{u}<\bar{u}$ in $\Omega \times(0, T-\epsilon)$. We then define

$$
U=\underline{u}-(2 k)^{-1}(\bar{u}-\underline{u}) .
$$

Simple computations show that

$$
\underline{u}>U \geq \frac{k+1}{2 k} \underline{u} \quad \text { in } \Omega \times(0, T-\epsilon)
$$

and

$$
\frac{2 k}{2 k+1} U+\frac{1}{2 k+1} \bar{u}=\underline{u} .
$$

It is clear that $f(x, t, v)=-a v+b(x, t) v^{p}$ is convex with respect to $v$ in $(0, \infty)$. Hence, by virtue of (3.13), we obtain

$$
f(x, t, \underline{u}) \leq \frac{2 k}{2 k+1} f(x, t, U)+\frac{1}{2 k+1} f(x, t, \bar{u}) .
$$

As a result, we have

$$
U_{t}-\Delta U=-\frac{2 k+1}{2 k} f(x, t, \underline{u})+\frac{1}{2 k} f(x, t, \bar{u}) \geq-f(x, t, U),
$$

from which and (3.12), we deduce

$$
\begin{cases}U_{t}-\Delta U \geq a U-b(x, t) U^{p} & \text { in } \Omega \times(0, T-\epsilon), \\ U=\infty & \text { on } \partial \Omega \times(0, T-\epsilon) \cup \bar{\Omega} \times\{0\} .\end{cases}
$$

Therefore, $U$ is a supersolution of $(2.1)$ and the comparison principle shows that $u_{n} \leq U$ in $\Omega \times(0, T-\epsilon)$ for all $n \geq 1$. Letting $n \rightarrow \infty$ we have $\underline{u} \leq U$, which is a contradiction with (3.12). Thus we must have $\underline{u}=\bar{u}$ and so the uniqueness conclusion holds. The proof is complete.

\section{REFERENCES}

[1] W. Al Sayed and L. Véron, On uniqueness of large solutions of nonlinear parabolic equations in nonsmooth domains, Adv. Nonl. Studies 9 (2009), 149-164.

[2] W. Al Sayed and L. Véron, Solutions of some nonlinear parabolic equations with initial blow-up, preprint (arXiv:0809.1805), 2008.

[3] C. Bandle, G. Díaz and J.I. Díaz, Solutions of nonlinear reaction-diffusion equations blowing up at the parabolic boundary (in French), C. R. Acad. Sci. Paris Sr. I Math. 318 (1994), 455-460.

[4] M. Chuaqui, C. Cortazar, M. Elgueta and J. Garcia-Melian, Uniqueness and boundary behavior of large solutions to elliptic problems with singular weights, Commun. Pure Appl. Anal. 3 (2004), no. 4, 653-662.

[5] Y. Du, Order Structure and Topological Methods in Nonlinear Partial Differential Equations, Vol. 1, Maximum Principles and Applications, World Scientific, Singapore, 2006.

[6] Y. Du, Asymptotic behavior and uniqueness results for boundary blow-up solutions, Diff. Integral Eqns. 17 (2004), 819-834. 
[7] Y. Du and Z.M. Guo, The degenerate logistic model and a singularly mixed boundary blow-up problem, Discrete Contin. Dyn. Syst. 14 (2006), 1-29.

[8] Y. Du and R. Peng, The periodic logistic equation with spatial and temporal degeneracies, Trans. Amer. Math. Soc., to appear.

[9] M. Marcus and L. Véron, The boundary trace of positive solutions of semilinear elliptic equations: the subcritical case, Arch. Ration. Mech. Anal. 144 (1998), 201-231.

[10] M. Marcus and L. Véron, Existence and uniqueness results for large solutions of general nonlinear elliptic equations, J. Evolution Eqns. 3 (2004), 637-652.

[11] M. Marcus and L. Véron, Maximal solutions of nonlinear parabolic equations with absorption, preprint (arXiv:0906.0669), 2009.

[12] P. Poláčik, P. Quittner and P. Souplet, Singularity and decay estimates in superlinear problems via Liouvilletype theorems. II. Parabolic equations, Indiana Univ. Math. J. 56 (2007), no. 2, 879-908. 